\title{
MUSÉE IMAGINAIRE: FONTANES GEMÄLDEGALERIE
}

\begin{abstract}
Musée imaginaire: Fontane's picture gallery
This paper tends to explore ,Fontane's picture gallery' referring to its genesis, characteristic and effect. In doing so the art critic Fontane is improving a distinctive image as an expert especially of English art und of the at that time in Germany leading Dusseldorf School of Painting (Düsseldorfer Malerschule). Furthermore it will be set out how the writer Fontane is acting in his work with pictures and pictorial perception. Eventually there is a suggestion for recovering ,Fontane's picture gallery' to make it possible for the contemporary audience to read Fontane's works in a wider and subtly differentiated manner.
\end{abstract}

KEY WORDS: Theodor Fontane - picture - visual memory - pictorial perception - English arts Dusseldorf School of Painting

Besaß Fontane eine Gemäldegalerie? Natürlich nicht, wird der Fontane-Kenner sagen, dazu reichten die Mittel bei weitem nicht; nur einige Stiche zierten sein Arbeitszimmer. Virtuelle Kunstsammlungen, wie sie sich heute dank des Internets jeder Kunstfreund in einer Größenordnung zulegen kann, die prinzipiell unbegrenzt ist, waren noch in weiter Ferne. Die technische Reproduzierbarkeit des Kunstwerks blieb an die Druckgrafik gebunden, die erst allmählich von der Fotografie abgelöst wurde. Die Begegnung mit dem gewünschten Bild fand in aller Regel vor dem Original (oder was dafür gehalten wurde) statt, angemessener Ersatz war schwer oder gar nicht zu haben, bei guten Stichen außerdem mit höheren Ausgaben verbunden. Kopien der begehrten Originale konnte sich nur leisten, wer wie der Kommerzienrat Ezechiel van der Straaten in dem Roman L'Adultera das Geld hatte, in Venedig einen Tintoretto in Auftrag zu geben (Fontane, 1990, S. 12-16; vgl. Neumann, 2001).

Fontanes Gemäldegalerie ist ein ,Museum im Kopf', das sich zumeist aus der direkten Begegnung mit Gemälden, Zeichnungen oder Skizzen speist. Musée imaginaire bezeichnet daher in diesem Fall ein durch die Erinnerungskraft gestütztes Bildgedächtnis, das sich im Laufe der Jahre und Jahrzehnte durch den Besuch von Museen, Galerien und Ausstellungen, aber auch von Privatsammlungen und Kunstsalons aufbaut und dann in unterschiedlicher Weise in Erscheinung tritt. Wir wissen 
von diesem imaginären Museum überhaupt nur, weil es bei dem Schriftsteller Fontane so vielfältig in Erscheinung tritt: in seinen Notiz- und Tagebüchern, Briefen, Reiseberichten, Kritiken und Romanen, selbst in solchen Texten, wo es am wenigsten zu erwarten ist, etwa in dem Bericht über seine Kriegsgefangenschaft 1870 in Frankreich. „Am fernen Horizont nach eben dieser Seite hin hing der gelbglühende Ball der Sonne und lieh allem ein entzückendes Licht; es war, als sähe man in eine der weitgedehnten Veduten Claude Lorrains" (Fontane, 1910, S. 56).

Bei solchen Überblendungen der wahrgenommenen Landschaft durch ein Landschaftsgemälde - sie kommen bei Fontane häufiger vor - werden Bilder aus dem Gedächtnis abgerufen, und auf dieses visuelle Gedächtnis konnte sich Fontane offenbar mehr verlassen als auf sein Namensgedächtnis: „Ich bin, was den Namen angeht, meiner Sache nicht sicher. Desto sicherer steht das Bild vor meinem Auge" (ebd., S. 31 f.). Aber, wird man an dieser Stelle fragen, handelt es sich nicht um einen Schriftsteller, der schon seiner Profession wegen zuerst und vor allem an der Arbeit seiner Kollegen interessiert sein musste?

Eine erste Überraschung erlebt man, wenn man sich einer wenig beachteten Folge biographischer Darstellungen zuwendet. Fontane hat sie für das 1862 in Leipzig erschienene Lexikon Männer der Zeit verfasst (Fischer, 2010). Nicht das Biographische überrascht, da es sich in vielerlei Formen durch sein gesamtes Werk zieht (Berbig, 2010). Die Überraschung liegt in der offenbar gezielten Auswahl jener Persönlichkeiten, denen er Kurzbiographien widmet. 23 behandeln Künstler und Architekten, auf Dichter entfallen nur sieben. In den teils mehrspaltigen Artikeln, die auf Leben, Werk, Wirkung, Stilrichtung und Bedeutung der Maler, Bildhauer und Baumeister eingehen, tritt einem ein Kunstkenner und Kunstkritiker entgegen, der augenscheinlich vieles aus eigener Anschauung kennt und überdies ein klares Urteil besitzt. Er ist in der damals in Deutschland führenden und international ausstrahlenden Düsseldorfer Malerschule (Baumgärtl, 2011) ebenso zu Hause wie in der zeitgenössischen bildenden und Baukunst Englands.

Gegenüber der Großgattung ,Biographie“ spielt die Kleingattung ,biographischer Artikel' die Rolle eines unscheinbaren Verwandten, der es selten zu einiger Aufmerksamkeit bringt (kein Verweis bei Grawe und Nürnberger, 2000; Nürnberger und Storch, 2007). Zu Unrecht; denn er legt ja nicht nur das Bild einer zeitgenössischen Persönlichkeit fest, er wirkt auch auf das Bild des Autors selbst zurück. Dieses differenziert, modifziert oder erweitert sich, je nachdem, welches Interesse und welches Wissen in dem jeweiligen Artikel zutage tritt. In der Vielzahl solcher Artikel zeigt es den Autor vielleicht sogar in einem ungewohnten Licht, und ungewohnt ist es, statt des Schriftstellers und Theaterkritikers den Kunstkritiker Fontane am Werk zu sehen.

Keine zweite Publikation spiegelt dieses Profil Fontanes in seinen mittleren Jahren so umfassend wider wie die Männer der Zeit mit dem Untertitel Biographisches Lexikon der Gegenwart. Neben der Historien-, Genre-, Landschafts-, Marine-, Porträt- 
und Tiermalerei finden die Bildhauerei, die Architektur und selbst die Karikatur ihren Platz. Noch der verdienstvolle Ausstellungsband Fontane und die bildende Kunst (Keisch, Schuster \& Wullen, 1998) ging an mehreren dieser Künstler vorüber, obwohl deren biographische Skizzen der Betrachtung wert sind - und wenn es nur der eine Satz über den Baumeister des Londoner Parlamentsgebäudes ist, denn in ihm finden sich alle Großbaumeister unserer Tage wieder, wenigstens in Berlin und anderen Großstädten Deutschlands: „Sir Charles Barry rechnete auf die Zustimmung der Nachwelt und hatte es längst aufgegeben, bei der Mitwelt, die freilich unter der Lästigkeit leidet, immer neue Millionen zahlen zu müssen, das Maß an Anerkennung zu finden, das ihm gebührt.“ (Männer der Zeit, Sp. 305). Man denkt hierzulande spontan an die Hamburger Elbphilharmonie oder das Berliner Schloss, von anderen Großbauten zu schweigen, und man blickt voller Respekt in das Nachbarland, wo eine Architekturikone wie die Philharmonie in Szczecin im Zeit- und Kostenrahmen fertiggestellt wurde.

Aber nicht nur an dem Baukünstler Barry, der bis heute durch seinen Westminster Palace berühmt ist, sondern auch an dem Historienmaler Edward Matthew Ward, der diesen Palast seit etwa 1855 mit Freskobildern schmückte (Männer der Zeit, Sp. 139 f.), hat der besagte Ausstellungsband kein Interesse genommen. Erstaunlich ist das schon deshalb, weil Fontane bei Ward eine feine Beobachtung zur Farbe macht:

In der Kunst des Abtonens, im Hervorbringen einer harmonischen Farbenwirkung ist er nicht immer glücklich, und während er nur allzusehr den Muth einer Farbe hat (er stellt z. B. das lange blutrothe Hemd der Charlotte Corday neben den hellblauen Seidenfrack Robespierre's u. dgl. m.), hat er doch das Geheimniß der Farbe nicht völlig belauscht. (Männer der Zeit, Sp. 140)

Fontane hat vieles in seinem Leben „mit durch die Kunst erzogenen Augen“ (Gadamer 1977, S. 41) gesehen. Sein Brief-, Reise- und Romanwerk bezeugt das in einem immer noch kaum erkannten Maße (vgl. Hoffmann, 2011; Fischer, 2012b, 2011, 2001). Die erste große Schule des Sehens war für ihn zweifellos England gewesen, wo er sich nach der Londoner Reise 1844 in den Jahren 1852 und 1855 bis Januar 1859 aufhielt. Beginnend mit dem Londoner Brief über die KunstAusstellung 1852 in der National Gallery (Fontane, 1979b, I, S. 220-225) und vorläufig endend mit der im Januar 1860 in Berlin gehaltenen Vorlesung über Englische Historienmalerei (Rasch, 2006, S. 426), hatte er sich vor allem in seinen Londoner Jahren immer wieder mit englischer Kunst, zumal mit englischer Gegenwartskunst, auseinandergesetzt, am intensivsten und fruchtbarsten wohl während der großen Art Treasures Exhibition 1857 in Manchester (Finke, 1998). Folglich konnte er tatsächlich in größerem Umfang aus eigener Anschauung (und Ausarbeitung) schöpfen, als er die englischen Künstler für das Lexikon Männer der Zeit porträtierte.

Woher aber hatte Fontane seine Kenntnisse der deutschen Gegenwartskunst, namentlich der Düsseldorfer Schule? Wir befinden uns in der Zeit vor seinen Berli- 
ner Ausstellungsberichten, die im Herbst 1862 einsetzen. Hier ist eine Quelle unmittelbarer Anschauung kaum gewürdigt worden, obwohl er selbst, freilich an versteckter Stelle, den entscheidenden Hinweis gibt. Er schließt die Biographie Theodor Hildebrandts, eines der gefeiertsten Maler dieser Schule, mit dem Hinweis: „Mehrere seiner besten Bilder befinden sich in der Galerie des Consul Wagner zu Berlin, die deshalb vorzugsweise Gelegenheit bietet, diesen Künstler, wie überhaupt die Düsseldorfer Schule zu studieren.“ (Männer der Zeit, Sp. 318). Diese Sammlung von 262 zeitgenössischen Gemälden des Bankiers und schwedisch-norwegischen Konsuls Johann Heinrich Wilhelm Wagener war, bevor sie als Legat der preußischen Krone vermacht wurde, um den Grundstock einer Nationalgalerie zu bilden, seit längerem öffentlich zugänglich. Nach dem Tod Wageners wurde sie am 22. März 1861 in den Räumen der Akademie der Künste aufgestellt und verblieb dort, bis sie 1876 in die neu erbaute Nationalgalerie, die heutige Alte Nationalgalerie, überführt wurde. Ein Umzug übrigens, der den zu diesem Zeitpunkt bereits völlig überforderten und alsbald seinen Abschied nehmenden Akademiesekretär Fontane zusätzlich belastete (Fischer, 2014, S. 292-325; Fischer, 1997, S. 33-35).

Wie oft in der Kunstgeschichte hat man sich erst mit geraumer Verspätung, in diesem Fall im Jahr 2011 anläßlich des 150. Jubiläums der Nationalgalerie, mit einer Ausstellung und einem Katalog des eigentlichen Begründers Wagener erinnert (Kittelman, Verwiebe \& Wesenberg, 2011). Er

\footnotetext{
trug in über vierzig Jahren die damals größte private Sammlung zeitgenössischer und internationaler Malerei in Berlin zusammen. Einen vergleichbaren Überblick über aktuelle Strömungen der bildenden Kunst gewährte um die Mitte des 19. Jahrhunderts lediglich die Sammlung des Grafen Athanasius von Raczyński. Wageners Sammlung war jedoch umfangreicher, vielfältiger und bürgerlicher. (Verwiebe, 2011, S. 321)
}

Sie war auf der Höhe der Zeit und Wagener selbst ein Freund und Förderer der Kunst und der Künstler in Berlin, München und Düsseldorf.

\footnotetext{
Während Raczyński mehr die nazarenische Kunst und ideale Figurenkompositionen bevorzugte, wandte sich Wagener der Landschafts- und Architekturmalerei, dem Genre und der Historie zu. Er profilierte sich als Förderer der Berliner Künstler seiner Zeit, als Liebhaber der sogenannten Münchner ,Kleinmeister', als Mäzen der Düsseldorfer Malerschule und als Verehrer der belgischen sowie der nach ihrem Vorbild entstandenen deutschen Historienmalerei.“(ebd., 2011, S. 321).
}

Nicht zuletzt dank seiner für das Publikum geöffneten Galerie in der BrüderstraBe und später auch in der Schillingsgasse entstand eine neue bürgerliche Kunstöffentlichkeit in Berlin. Mehr als ein Viertel der Sammlung machten Gemälde der Düsseldorfer Schule aus.

Von den für das Lexikon Männer der Zeit porträtierten Malern gehörten Andreas und Oswald Achenbach, Wilhelm Camphausen, Hans Gude, Theodor Hildebrandt, Caspar Scheuren und Adolf Tidemand ganz, Hermann Stilke und Teutwart 
Schmitson vorübergehend der Düsseldorfer Schule an. Aus der Wagenerschen Galerie waren Fontane Andreas Achenbach mit Herbstliche Waldlandschaft (Kittelmann et al., 2011, S. 1 u. Abb. 1), Camphausen mit dem Historienbild Cromwell'sche Reiter (ebd., S. 19 u. Abb. 34), Hildebrandt mit dem historischen Genrebild Der Krieger und sein Kind (ebd., S. 47 u. Abb. 90) und Stilke mit dem Historienbild Raub der Söhne Eduards (ebd., S. 132 f. u. Abb. 239) bekannt. Teutwart Schmitson hat er offenbar in seinem Berliner Atelier aufgesucht: „(...) und der Besuch seines Ateliers gehört zu den besonders angenehmen, indem eine große Fülle angefangener und halb vollendeter Gemälde von allen Dimensionen einen vollen Blick in die Darstellungswelt und Schöpfungsweise des Künstlers zulassen.“" (Männer der Zeit, Sp. 310). Für einzelne biographische Artikel, so im Fall Oswald Achenbachs, hat Fontane auf die Bilder der Berliner Kunstausstellung 1860 zurückgegriffen. Mit Sicherheit dürfte er außerdem die Ravenésche Bildergalerie und die Galerie des Berliner Kunstvereins für seine Zwecke genutzt haben. Hier waren u. a. Bilder von Caspar Scheuren sowie von Hans Gude und Adolf Tidemand zu sehen. Nicht zu vergessen sind Fontanes Besuche in Sachse's Kunstsalon in den sechziger Jahren. Louis Friedrich Sachse war einer der

Begründer des modernen Kunsthandels in Berlin. Er gab Künstlern die willkommene Gelegenheit, ihre Werke in seinem Kunstsalon und später in seiner Permanenten Gemäldegalerie zu präsentieren und übte durch Vermittlung und den Verkauf der Arbeiten vor allem deutsch- und französischsprachiger Maler wesentlichen Einfluss auf das Kunstgeschehen und die Kunstentwicklung in der preuBischen Hauptstadt aus. (Nerlich \& Savoy, 2013, S. 250)

Warum diese detaillierten Angaben (sie sind lediglich beispielhaft)?

Wir brauchen nicht nur eine Bibliothek der Bücher, die Fontane gelesen hat (Rasch, 2005), wir brauchen auch ein Inventar der Bilder, die Fontane gesehen hat, denn wir gehen, wenn wir seine Texte lesen, durch ein Musée imaginaire. Das beginnt bereits in diesen Artikeln. Da ist ein ständiges Vergleichen zwischen Malern des einen wie des anderen Genres, der einen wie der anderen Schule, zwischen deutschen und englischen Künstlern, der englischen Künstler untereinander - das bei Sir Edwin Landseer, dem berühmtesten Tiermaler seiner Zeit, zum köstlichen Bonmot wird.

Nur Thomas Lawrence, dessen Blüthezeit als Porträtmaler in die zwanziger Jahre dieses Jahrhunderts fällt, ist in ähnlich glänzender Weise honorirt worden, wiewohl sich, auch im Vergleiche mit ihm, dem aristokratischen Porträtmaler par excellence, die heitre Thatsache ergiebt, daß der englische Adel bereit gewesen ist die Porträts seiner Lieblingshunde glänzender zu bezahlen als seine eignen. (Männer der Zeit, 1862, Spalte 225 f.)

Bis 1870, bis zu seinem Eintritt in die Vossische Zeitung als Theaterkritiker, zeichnet sich im Jahrzehnt davor ebenso klar das Profil des Kunstkritikers Fontane in Ausstellungsberichten und Buchrezensionen ab, die auch danach noch nicht ver- 
siegen. Von seinem aufschlussreichen Fragment einer Carl Blechen-Biographie gar nicht zu reden (Streiter-Buscher, 2010). Aus dem CEuvre dieses für seine Zeit ungewöhnlichen und gerade deshalb faszinierenden Malers hätte Fontane gerne für sich persönlich, für seine „Galerie“, seinen „Salon“ und sein „Wohnzimmer“, eine Reihe großer und kleinerer Bilder ausgewählt. (Streiter-Buscher, 2010, S. 157-168) Blieb auch diese Gemäldegalerie ein imaginäres Museum, so sagt sie doch einiges über seine Vorlieben und Wünsche, nicht zuletzt über sein differenziertes Kunsturteil aus. Blechens Gemälde Das Semnonenlager ist überdies als „historische Landschaft“ in Fontanes Wanderungen durch die Mark Brandenburg eingegangen; er schreibt dessen Stimmung, Details und Gestalten vermöge der „künstlerische[n] Phantasie“ der gegenwärtigen märkischen Landschaft ein (Fontane, 1977, S. 553-558). Auch in diesem Fall wendet er die Technik der Überblendung an, besser gesagt, er nimmt sie vorweg, denn er verbindet wie heutzutage die Medien Film und Fernsehen zwei Bildereignisse derart, dass das eine kontinuierlich in das andere übergeht.

Fontane ist in Kunstangelegenheiten nicht nur durch eine „Schule des Sehens“, sondern auch durch eine „Schule der Urteilsbildung“ gegangen, und in dieser Hinsicht spielte der Rütli, eine Berliner Gruppierung von Künstlern, Schriftstellern und Kunsthistorikern, eine wichtige Rolle. So schrieb er an den Schriftstellerkollegen Paul Heyse im Dezember 1860:

\footnotetext{
Es ist wirklich nur Pflicht einzuräumen, daß ich noch niemals einem Kreise angehört habe, in dem durch die Friktion der Geister so viel Anregung gegeben, so viel Leben und Wärme producirt worden wäre. Gespräche über Kunst, namentlich über gewisse kitzliche, eben jetzt in Streit befindliche Punkte, wie sie zwischen Blomberg, Menzel, Lazarus, Lübke und Lucae (bei architektonischen Fragen) mit Eifer und Sachkenntniß, und namentlich ohne alle Verranntheit und Stagnation geführt worden sind, kommen gewiß nicht allzu häufig vor. Es trifft sich, daß man einem famosen Kerl auf diesem oder jenem Gebiet begegnet und von ihm lernt, aber einer Vielheit tüchtiger Kräfte, einer reichen Nüancirung wohlberechtigter und wohlverteidigter Anschauungen begegnet man selten." (Fontane, 1979a, S. 18)
}

Fontanes zum Teil langjährige und intensive Beziehungen zu Künstlern wie Adolph Menzel, Hugo von Blomberg und August von Heyden, zu namhaften Kunsthistorikern wie Friedrich Eggers, Franz Kugler, Wilhelm Lübke und Carl Schnaase, aber auch zu Architekten wie Richard Lucae sind bei der Klärung seiner Kunstkenntnis und der Bildung seines Kunsturteils unbedingt in Anschlag zu bringen. „Ich habe mein Leben unter Malern verbracht", schreibt Fontane im hohen Alter, 1895, pointiert an Maximilian Harden, ,und ich darf sagen, daß ich eine große Kunstkenntniß habe“" (Fontane, 1982, S. 511). Das hat Folgen für sein Werk gehabt und kann deshalb für den Leser nicht ohne Folgen bleiben.

Lässt man Fontanes Romane Revue passieren, stellen sich bei genauer Lektüre Bilder in Fülle ein. Dem Leser geht es wie der Romanfigur, die sich des Erlebten erinnert: „Hundert Bilder (...) zogen an ihm vorüber (...)“ (Fontane 1990, S. 245). Und wenn es nicht gleich „hundert“ sind in einem Roman, so können es doch neben 
den tatsächlich aufgerufenen und näher beschriebenen Bildern Anspielungen oder Andeutungen sein, die im Kopf des Lesers visuelle Reflexe auslösen und sich daraufhin zu Bildern verdichten. Das macht die spezifische ,Bildhaftigkeit' von Fontanes Romanen aus, wobei die individuelle Einbildungskraft des Lesers natürlich stets mitwirkt bei deren mentaler Realisierung. Die Forschung hat sich solcher Fragen erst mit einiger Verspätung angenommen. Dann aber hat sie mit kunsthistorischem Gespür aus Effi Briest „ein Leben nach christlichen Bildern“ herausdestilliert (Schuster, 1978), in L'Adultera die aufschlussreichen „Bildergespräche“ analysiert (Jung, 1991) und in Cécile „Bilder als Merkmale kritischen Erzählens“ dechiffriert (Jung, 1990). Es scheint, als hätte die steigende Bedeutung der visuellen Kultur in unserer Zeit auch eine zunehmende Sensibilisierung für die sprachliche Organisation visueller Prozesse bewirkt. Das betrifft vor allem die späteren Romane, in denen das Bildliche selbst thematisch wird, wie in L'Adultera, Cécile und Die Poggenpuhls, oder in denen das Bildliche dem aufmerksamen Leser regelrecht ins Auge springt, wie in Effi Briest. Das ist zuletzt noch einmal an der gleichnamigen weiblichen Hauptfigur des Romans im Rückgriff auf Gemälde des von Fontane besonders geschätzten Präraffaeliten John Everett Millais sowie auf verschiedene Punch-Zeichnungen seines Freundes George du Maurier dargestellt worden (Fischer, 2012a).

Indessen zeigt sich bei näherer Betrachtung, dass solche visuellen Geschehnisse sowie eine bildhafte Wahrnehmung bereits in Vor dem Sturm eine ,unübersehbare' Rolle spielen, ja, dass in den voraufgegangenen Bänden der Wanderungen Muster der Bildlichkeit sich finden, die in diesem ersten Roman Fontanes wiederkehren (Fischer, 2014, S. 17-57). In Vor dem Sturm reicht die zitathafte Anlehnung an die Bilderwelt jedoch weiter, von den Fresken des Camposanto in Pisa (Steland, 2006) über Giulio Romano, Tintoretto und Joshua Reynolds (Fontane, 2011, II, S. 31, 135) bis zu Claude Lorrain:

Die Sonne glühte hinter den Bäumen, und je nachdem die Lichter fielen, schimmerte das braune Laub der Eichen golden oder kupferfarben, während die schwarzen Tannenwipfel wie scharfgezeichnete Schatten in der schwimmenden Glut des Abends standen. [...] Bummcke entsann sich, selbstverständlich von Kopenhagen her, eines dieselben Abendtöne wiedergebenden Claude-Lorrain. (Fontane, 2011, II, S. 194)

Es gab in der Königlichen Gemäldesammlung in Kopenhagen tatsächlich ein Landschaftsgemälde dieses wirkmächtigsten Meisters aller Landschaftsmalerei (Thaarup, 1828, S. 163).

Dort hatte sich Fontane 1864 auch mit der „dänischen Malerschule“ (deren Eigentümlichkeit er jedoch bezweifelte), besonders mit Jens Juel und Nicolai Abildgaard, vertraut gemacht (Fontane, 2004, S. 36-45).

Weitet man die Betrachtung auf die englisch-schottische Reiseliteratur und die sie begleitenden Zeugnisse der vierziger und fünfziger Jahre aus, gelangt man rasch zu der Feststellung, dass schon beim Übergang von der Balladendichtung zur Prosa 
das ,Bild“ in vielerlei Gestalt eine prägnante Funktion erhält (Fontane 1979b, I, S. 72, 79-81 u. ö.; II, S. 358-360, 367-381 u. ö.). Kunstkritik, zum Teil sehr prononcierte, findet sich dort bereits ebenso wie eine durch das Massenmedium Panorama (Kunst- und Ausstellungshalle der Bundesrepublik Deutschland, 1993; Oettermann, 1980) oder die Camera obscura (Crary, 1996, S. 37-73) vorgeprägte Wahrnehmungskonvention:

(...) blicken wir jetzt, den Rücken fest ans Geländer gelehnt, über Menschen, Brücke und Brandung hinweg, bis hin auf den prächtigen Brighton-Kai, dessen durch Entfernung verkleinertes Treiben nun wie ein reizendes still bewegtes Camera-obscura-Bild vor uns liegt. (Fontane 1979b, I, S. 337)

Dieses ,still bewegte“ Bild wird vor dem Auge des Lesers in seinen charakteristischen Einzelzügen entfaltet, als nähme dieser selbst es wahr:

Damen zu Pferde in schwarzem, wallendem Reithabit galoppieren vorüber, reizend gekleidete Kinder in ihrer Ziegenbockequipage fahren auf und ab, breitschultrige Fischergestalten mit Teerjacke und Krempenhut winden das heimkehrende Boot aus der Brandung ans sichre Ufer - Leben überall, aber das stille Leben eines Bildes: kein Mißklang unterbricht den Zauber, dem Aug und Seele hingegeben sind.“( (ebd.)

Stärker bewegte Bilder erzeugt Fontane hingegen in Analogie zum damals beliebten Moving Panorama, das bei dem Zuschauer die Illusion der Selbstbewegung durch die Landschaft hervorruft (Fischer, 2014, S. 26 f.).

Nicht allein die virtuelle Realität der Romane oder die Kunstprosa der Reisefeuilletons bezeugt die zu Bildern gerinnende Wahrnehmung von Raum, Architektur, Mensch und Landschaft; auch in den autobiographischen Schriften und Briefen wird man „diese[r] von Fontane immer wieder beschworene[n] Bildtendenz visueller Prozesse“ (Wullen 1998, S. 260) gewahr. „Ich werde das Bild, das ich hier sah“, schreibt er in Aus den Tagen der Occupation, ,nicht leicht vergessen. Die Engländer, in ihren historischen Bildern, behandeln mit Vorliebe die ,kleine Puritanerfamilie', die in Haus oder Hütte ihre Abendmahlzeit nimmt (...). So war es auch hier“" (Fontane, 1872, S. 273). Solche bildanaloge Wahrnehmungsweise findet sich in demselben Reisebericht auch in Form einer Rahmenschau:

Wie es aber keine Armuth giebt, die nicht irgend einen Reichthum einschlösse, so war auch hier für einen solchen gesorgt, und dieser Reichthum war - das Fenster. Es hatte nur die Größe einer Luke, stand auf und wirkte lediglich wie der Rahmen für eine Landschaft, in die ich hineinsah: erst Gärten, dann Dächer und scheinbar unmittelbar dahinter die hohen Waldwände der Vogesen. Die Sonne ging unter. Schmetterlinge flogen im goldnen Abendlichte aus und ein. (ebd., S. 265 f.)

Um Aus den Tagen der Occupation ein letztes Beispiel zu geben, diesmal von einer herausragenden Architektur, der Kathedrale von Metz:

(...) sie hat sich dem Auge eingeprägt, um der Erinnerungen halber, die jene Zeit ausfüllen, aber auch um ihrer Schönheit willen. ,Das großartigste Werk des französisch-gothischen Kirchenbaus in Lothringen', - so schreibt W. Lübke in seiner Architekturgeschichte.“ (ebd., S. 161). 
Zweierlei ist daran zu bemerken: Dass die affektive Qualität des Bildes die Erinnerungskraft an das Bild steigert, und dass diese zweitens durch Lübkes Geschichte der Architektur eine Bestätigung erhält. Anschauung, Affekt und ästhetisches Urteil fließen ineinander. Fontane hatte das reich illustrierte Werk des befreundeten Kunsthistorikers einige Jahre zuvor rezensiert.

Insbesondere die Langzeitwirkung von Bildern hängt entscheidend von den Umständen, unter denen sie wahrgenommen werden, sowie von der Stärke der Affekte ab, mit der sie behaftet sind. In seiner Autobiographie Von Zwanzig bis Dreißig erinnert sich Fontane einer jungen Diakonissin, die unversehens aus dem Rahmen tritt: ,[Sie] glich in ihrem schlichten rotblonden Haar und den großen Kinderaugen einem aus dem Rahmen herausgetretenen Präraffaelitenbilde" (Fontane, 1980, S. 354). Kein Zweifel, hier ruft Fontane nach vierzig Jahren John Everett Millais' Autumn Leaves aus dem Bildgedächtnis ab, ein Gemälde, das ihn von allen Präraffaelitenbildern am stärksten beeindruckt hat (Keisch et al., 1998, S. 102 f.). Es ist deshalb auch kein Zufall, dass bis in seinen letzten Roman, Der Stechlin, die Präraffaeliten ein Thema sind (Fischer, 2011, S. 378-384).

Auf das Landschaftliche und Stadtlandschaftliche hin angesehen, sind die Beispiele dieses bildgebenden Verfahrens so zahlreich, dass Andeutungen genügen müssen. In Kriegsgefangen, immerhin auf einem Gefangenentransport, gipfelt die Beschreibung eines ,entzückende[n] Stückes Natur, das ich vielleicht am besten als das ,Land um Vichy“ bezeichne“, in dem Satz: „Dazu hatte alles einen satten, braungrünen Ton, der mich mehr als einmal an Ruysdael erinnerte, von dem ich vier Wochen vorher einiges Treffliche in Nancy gesehen hatte" (Fontane, 1910, S. 64f.). Die im Musée des Beaux-Arts in Nancy betrachteten Landschaftsgemälde Jacob van Ruisdaels fließen im Ton in das wahrgenommene Landschaftsbild ein. Bilderlebnis und Landschaftserfahrung verbinden sich. Besançon wird in Kriegsgefangen deshalb zu einem „reizende[n] Bild“, weil es selbst dem ästhetischen Prinzip des Malerischen entspricht:

\footnotetext{
Alle charakteristischen Städte wirken viel anheimelnder als die architektonisch korrekten; ja die malerische Schönheit - ich erinnere nur an Kopenhagen - ist so entschieden siegreich über die bauliche, daß wir zuletzt jede Stadt schön nennen, die wie ein reizendes Bild uns berührt. (Fontane, 1910, S. 31)
}

Stadtbilder können sogar in direkter Analogie zu Landschaftsgemälden berühmter Künstler wahrgenommen werden: „Der vorherrschende Charakter, zumal bei entsprechender Beleuchtung, möchte in der Wirklichkeit kaum etwas Analoges finden; Oxford, in einer gewissen Entfernung gesehen, liegt da wie eine Landschaft Poussins oder Claude Lorrains." (Fontane, 1979b, I, S. 580). Das schrieb Fontane 1860; acht Jahre früher hatte er die beiden Maler als „,neue Meister“ in der National Gallery in London kennengelernt (ebd., S. 122). Aufgrund der vorhandenen Quellen, nicht zuletzt auch der Tagebücher seiner italienischen Kunstreisen 1874 und 1875, 
lässt sich bis an das Lebensende recht gut rekonstruieren, welche Bilder Fontane wann gesehen hat, so dass der Aufbau des besagten Musée imaginaire ziemlich genau verfolgt werden kann.

Bei anderer Gelegenheit wurde gezeigt, wie Fontane die Auseinandersetzung mit der Kunst in seinen Romanen konversationell weitertreibt bis zu den Großstadtimpressionen Franz Skarbinas und den von den französischen Impressionisten wiederentdeckten japanischen Farbholzschnitten mit ihren frappanten Naturausschnitten (Fischer, 2001, S. 115-117). Daran wird erkennbar, dass das imaginäre Museum kein angestaubtes, sondern ein lebendiges, sich über die Jahre und Jahrzehnte veränderndes Museum ist. So dient es in den Romanen auch dazu, aktuelle Kunstgespräche zu initiieren. Sie stellen im Stechlin, Fontanes letztem Roman, eine facettenreiche Reflexion der Beziehungen zwischen Kunst und Gesellschaft dar und bilden eine „immanente Ästhetik seines Gesellschaftsromans“ (Fischer, 2011, S. 387).

Aber kennt der Durchschnittsleser noch die Bilderwelt, in der Fontane und sein Publikum lebten? Sie ist zum größten Teil aus den Museen verschwunden und daher nicht mehr Element seiner Erfahrungswelt. Der von der Anspielung oder bloßen Chiffre überspringende Funke, der das Bildgedächtnis der Zeitgenossen erhellte, trifft heute weitgehend ins Leere. Damit geht aber auch eine wichtige Dimension der Lektüre verloren, die im Wechselspiel von Wort und Bild von oft großem Reiz und subtiler Raffinesse ist. Um eine solche differenzierte Lektüre der Romane wiederzugewinnen, müssten die alten Bilder aus der Latenz treten, müssten erkannt, dechiffriert, dokumentiert und, wenn möglich, digitalisiert werden, damit sie für den Lektüreprozess bereitstehen und wieder abgerufen werden können - zwar nicht aus dem Gedächtnis, aber in einer Bildleiste parallel zum Text auf einem E-Book. Da Fontane „mit Bildern erzählt“ (Fischer, 2001), könnte das Ergebnis ebenso überraschend wie anregend für weitere Lektüren sein.

\section{Bibliographie}

Baumgärtl, B. (Hrsg.). (2011). Die Düsseldorfer Malerschule und ihre internationale Ausstrahlung 1819-1918. 2 Bde. Petersberg: Imhof.

Berbig, R. (Hrsg.). (2010). Fontane als Biograph. Berlin: De Gruyter.

Crary, J. (1996), Techniken des Betrachters. Sehen und Moderne im 19. Jahrhundert (A. Vonderstein, Übers.). Dresden: Verlag der Kunst.

Finke, U. (1998). ,... ein Musterplatz für die gesamte moderne Kunst“. Die Art Treasures Exhibition in Manchester. In C. Keisch, P.-K. Schuster \& Moritz Wullen (Hrsg), Fontane und die bildende Kunst (S. 292-302). Berlin: Henschel

Fischer, H. (2014). Märkisches und Berlinisches. Studien zu Theodor Fontane. Berlin: Stapp.

Fischer, H. (2012a). „Effie“ und „Effi“ - Versuch über einen Namen mit neuen Zeugnissen in Bild und Text. Fontane Blätter, 94, 22-47.

Fischer, H. (2012b). Rezension Photographie, Malerei und visuelle Wahrnehmung bei Theodor Fontane von N. Hoffmann. Germanistik, 53, 576. 
Fischer, H. (2011). Zwischen Kunst, Kirche und Cornelius. Zwei wenig beachtete Künstlerbegegnungen Fontanes und ihre Spuren im Werk. Wirkendes Wort, 61, 363-387.

Fischer, H. (2010). „Männer der Zeit““. Fontanes biographische Artikel für Carl B. Lorck. In R. Berbig (Hrsg.), Fontane als Biograph (S. 187-204). Berlin: De Gruyter.

Fischer, H. (2001). „Gemmenkopf“ und „Nebelbild“. Wie Fontane mit Bildern erzählt. In T. Mehigan \& G. Sauder (Hrsg.), Roman und Ästhetik im 19. Jahrhundert. Festschrift für Christian Grawe zum 65. Geb. (S. 109-137). St. Ingbert: Röhrig.

Fischer, H. (1997). „... so ziemlich meine schlechteste Lebenszeit“. Unveröffentlichte Briefe von und an Theodor Fontane aus der Akademiezeit. Fontane Blätter, 63, 26-47.

Fontane, Th. (1872). Aus den Tagen der Occupation. Eine Osterreise durch Nordfrankreich und ElsaßLothringen (2. Aufl.). 2 Bde. Berlin: Verlag der Geheimen Ober-Hofbuchdruckerei (R. v. Decker).

Fontane, Th. (1910). Kriegsgefangen. Erlebtes 1870 (Hochschulausgabe). Berlin: F. Fontane \& Co.

Fontane, Th. (1977). Wanderungen durch die Mark Brandenburg. Werke, Schriften und Briefe II/2 (2. Aufl.). W. Keitel \& H. Nürnberger (Hrsg.). München: Hanser.

Fontane, Th. (1979a). Briefe. Werke, Schriften und Briefe IV/2. O. Drude, G. Krause \& H. Nürnberger (Hrsg.). München: Hanser.

Fontane, Th. (1979b). Wanderungen durch England und Schottland. 2 Bde. H.-H. Reuter (Hrsg.). Berlin: Verlag der Nation.

Fontane, Th. (1980). Von Zwanzig bis Dreißig. W. Keitel (Hrsg.). Frankfurt a. M.: Ullstein.

Fontane, Th. (1982). Briefe. Werke, Schriften und Briefe IV/4. O. Drude \& H. Nürnberger (Hrsg.). München: Hanser.

Fontane, Th. (1990). L'Adultera. Cécile. Werke, Schriften und Briefe I/2 (3. Aufl.). H. Nürnberger (Hrsg.). München: Hanser.

Fontane, Th. (2004). Mein skandinavisches Reisebuch - Reisen durch Dänemark, Jütland und Schleswig. C. Andree (Hrsg.). Kiel: Walter G. Mühlau.

Fontane, Th. (2011). Vor dem Sturm. Roman aus dem Winter 1812 auf 13. 2 Bde. C. Hehle (Hrsg.) Berlin: Aufbau.

Gadamer, H.-G. (1977). Die Aktualität des Schönen. Kunst als Spiel, Symbol und Fest. Stuttgart: Reclam.

Grawe, C., \& Nürnberger, H. (Hrsg.). (2000). Fontane-Handbuch. Stuttgart: Kröner.

Hoffmann, N. (2011). Photographie, Malerei und visuelle Wahrnehmung bei Theodor Fontane. Berlin: De Gruyter.

Jung, W. (1991). Bildergespräche. Zur Funktion von Kunst und Kultur in Theodor Fontanes ,L'Adultera“. Stuttgart: M \& P Verlag für Wissenschaft und Forschung.

Jung, W. (1990). „Bilder und immer wieder Bilder ...“ Bilder als Merkmale kritischen Erzählens in Theodor Fontanes „Cécile“. Wirkendes Wort, 40, 197-208.

Keisch, C., Schuster, P.-K., \& Wullen, M. (Hrsg.). (1998). Fontane und die bildende Kunst. Berlin: Henschel.

Kittelmann, U., Verwiebe, B., \& Wesenberg, A. (Hrsg.). (2011). Die Sammlung des Bankiers WagenerDie Gründung der Nationalgalerie. Berlin: Stiftung Preußischer Kulturbesitz/E. A. Seemann.

Kunst- und Ausstellungshalle der Bundesrepublik Deutschland (Hrsg.). (1993). Das Panorama als Massenunterhaltung des 19. Jahrhunderts. Basel/ Frankfurt a. M.: Stroemfeld/Roter Stern.

Männer der Zeit. (1862). Biographisches Lexikon der Gegenwart. Mit Supplement: Frauen der Zeit. Leipzig: Carl B. Lorck.

Nerlich, F., \& Savoy, B. (Hrsg.). (2013). Pariser Lehrjahre. Ein Lexikon zur Ausbildung deutscher Maler in der französischen Hauptstadt. Bd. 1. 1793-1843. Berlin: De Gruyter.

Neumann, G. (2001). Speisesaal und Gemäldegalerie. Die Geburt des Erzählens aus der bildenden Kunst: Fontanes Roman „L'Adultera“. In T. Mehigan \& G. Sauder (Hrsg.), Roman und Ästhetik im 19. Jahrhundert (S. 139-169). St. Ingbert : Röhrig , 2001. 
Nürnberger, H., \& Storch, D. (Hrsg.). (2007). Fontane-Lexikon. Namen - Stoffe - Zeitgeschichte. München: Hanser.

Oettermann, St. (1980). Das Panorama. Die Geschichte eines Massenmediums. Frankfurt a. M.: Syndikat.

Rasch, W. (2005). Zeitungstiger. Bücherfresser. Die Bibliothek Theodor Fontanes als Fragment und Aufgabe betrachtet. Imprimatur, 19, 103-144.

Rasch, W. (2006). Theodor Fontane Bibliographie. Werk und Forschung. 3 Bde. E. Osterkamp \& H. Delf von Wolzogen (Hrsg.). Berlin: Walter de Gruyter.

Schuster, P.-K. (1978). Theodor Fontane: „Effi Briest“ - Ein Leben nach christlichen Bildern. Tübingen: Niemeyer.

Steland, D. (2006). Memento mori. Ein kryptisches Zitat und seine epische Integration in Theodor Fontanes „Vor dem Sturm“. Fontane Blätter, 81, 46-79.

Streiter-Buscher, H. (2010). Die nichtvollendete Biographie. Theodor Fontanes „Karl Blechen“-Fragment. In R. Berbig (Hrsg.), Fontane als Biograph (S. 133-172). Berlin: De Gruyter.

Thaarup, F. (1828). Kopenhagen mit der Umgegend; besonders zum Nutzen der Fremden und als Anleitung für sie. Kopenhagen: Edward Ludwig Thaarup.

Verwiebe, B. (2001). „Der Erste, der den wahren, neu belebten Genius erkannte“ - Die Düsseldorfer Malerschule und ihr Sammler Joachim Heinrich Wilhelm Wagener. In B. Baumgärtl (Hrsg.), Die Düsseldorfer Malerschule und ihre internationale Ausstrahlung (S. 321-327). Petersberg: Imhof.

Wullen, M. (1998). Über das Sehen bei Fontane. In C. Keisch, P.-K. Schuster \& M. Wullen (Hrsg.), Fontane und die bildende Kunst (S. 257-261). Berlin: Henschel. 